\title{
Designing Messenger Visual, an Instant Messaging Service for Individuals with Cognitive Disability
}

\author{
Pere Tuset ${ }^{1}$, Juan Miguel López ${ }^{2}$, Pere Barberán ${ }^{1}$, Léonard Janer ${ }^{1}$, \\ and Cristina Cervelló-Pastor ${ }^{3}$ \\ ${ }^{1}$ Secció de Projectes de Transferència de Coneixement, TecnoCampus Mataró-Maresme, \\ Av. Ernest Lluch 32, 08302 Mataró, Spain \\ \{ptuset, barberan, leonard\} @ tecnocampus. cat \\ ${ }^{2}$ Departamento de Lenguajes y Sistemas Informáticos, Universidad del País Vasco, \\ C/Nieves Cano 12, 01006 Vitoria-Gasteiz, Spain \\ juanmiguel.1opez@ehu.es \\ ${ }^{3}$ Departament d'Enginyeria Telemàtica, Universitat Politècnica de Catalunya, \\ Av. Esteve Terradas 7, 08860 Castelldefels, Spain \\ cristina@entel.upc.edu
}

\begin{abstract}
Considering the importance of Internet-based communications in our society, the lack of Instant Messaging (IM) services adapted to individuals with cognitive disabilities who have difficulties using written language creates a situation of exclusion that has a negative impact on their daily lives. To alleviate this situation we present Messenger Visual, an IM service that uses pictograms as the main communication system. Along the paper we introduce the main design aspects of an IM service to support pictogram-based communications, as well as the design and evaluation aspects of an IM client that takes into account both the pictogram-based communication and the user interface accessibility requirements of individuals with cognitive disabilities.
\end{abstract}

Keywords: instant messaging services, augmentative and alternative communication, cognitive disability, user-centered design.

\section{Introduction}

Almost ten percent of the world's population lives with some type of disability [1]. One common aspect of disabled people is that they are prone to be excluded from society due to their condition. And despite the efforts being made to bridge the gap the exclusion situation is still far from being optimal, especially if we consider the digital society we are embracing. Today, albeit the shift from analog to digital technology has eliminated some accessibility barriers [2], individuals that live with a disability are also left apart from the digital society because most technology still fails to meet their accessibility requirements [3]. This situation needs to be addressed, as it reduces the independence of disabled individuals and causes social isolation.

Individuals with cognitive disabilities are one prime example of digital exclusion. Due to their condition they suffer from attention, memory and language impairments which difficult daily activities such as task planning and information processing [4]. 
But despite most of them are capable of using technological devices [5], the software that enables access to Internet services, such as browsers to access the World Wide Web (WWW), fails to take their requirements into account. For instance, the user interface of mainstream web browsers uses abstract concepts like tabs that are not easy to understand for such users. And other Internet services, such as electronic mail or instant messaging, are not in a better shape if we take into consideration that information in such media is usually presented in a textual format.

Nevertheless, this situation can be improved by developing user-centric software that takes into account the requirements of individuals with cognitive disabilities. This enables them to participate in the digital society [6], thus improving their independence and reducing social exclusion. For instance, the WWAAC (World Wide Augmentative and Alternative Communication) project [7] developed a WWW browser that uses pictogram-based Alternative and Augmentative Communication (AAC) to represent the information. But, to our knowledge, nowadays there is no single Instant Messaging (IM) service that meets the communication and user interface requirements of individuals with cognitive disabilities.

In this paper we present Messenger Visual, an IM service that enables individuals with cognitive disability to communicate over the Internet. The main differential aspect of Messenger Visual is that it replaces textual messages with pictograms-based messages, while providing the basic functionality of an IM service. We have also developed an IM client that takes into account the user interface accessibility requirements of individuals with cognitive disabilities. Finally, we have also evaluated the IM client using a user-centered approach, where users with cognitive disability have tested Messenger Visual as a regular activity.

The rest of the paper is organized as follows. Section 2 provides background related to the topics of the paper, namely Instant Messaging and Augmentative and Alternative Communications. Section 3 presents the design of a pictogram-based IM service to enable people with cognitive disability to communicate over the Internet. Section 4 presents the design of a pictogram-based IM client using a user-centered approach. Finally, Section 5 states the conclusions outlined from the development and evaluation of the project, and identifies the work that remains to be done in the future.

\section{Related Work}

IM services [8] are designed to enable users to exchange near real-time presence information and text-based messages over a public network, usually the Internet, to communicate with their contacts. In general, IM services rely on a client-server architecture to operate; IM servers provide features such as user access control and message routing, whereas IM clients provide a graphical interface for users to communicate. Nevertheless, IM services have evolved and now provide many other features besides the ones used for text-based communication, such as audio and video conferencing, file transfers and shared desktop.

Today most IM services are run by Internet-based companies, such as AOL, Yahoo and Microsoft. The protocols that support such IM services, as well as the software that enables users to communicate, are proprietary and their specifications are not publicly available. To provide with an open alternative the IETF 
(Internet Engineering Task Force) had different groups dedicated to Internet-based IM services, obtaining a general model which was later adopted by two IM protocols, namely SIMPLE (SIP for Instant Messaging and Presence LEveraging) and XMPP (eXtensible Messaging and Presence Protocol). SIMPLE provides a set of extensions to SIP (Session Initiation Protocol) to support IM services, whereas XMPP derives from Jabber, an IM protocol based on XML (eXtensible Markup Language) which was initially designed by the open-source community [9].

Augmentative and Alternative Communications (AAC) [10] is a set of methodologies aimed at complementing or replacing written or spoken communications for those individuals that have such abilities impaired, either temporarily or permanently, due to injury or illness. One form of AAC are pictogram-based communication systems, which are built upon drawings or images that represent real objects or abstract concepts to enable individuals sharing internal states, feelings, ideas and experiences. Communication using pictograms usually consists of individuals selecting elements from a set of possibilities with the aid of an Assistive Technology (AT), which can either be low or high technology (i.e. a cardboard, a personal communicator or a computer).

Nowadays, there are many different pictogram-based communication systems, which are usually classified according to the transparency level of its pictograms; the level of resemblance between the pictograms and the objects or concepts they represent [10]. For instance, Rebus is a pictogram-based language developed by Woodcok to teach reading to children. PIC (Picture Ideogram Communication) was developed by Maharaj and its main characteristic is that they have a reverse contrast, i.e. white on black. Blissymbols was developed by Karl Blitz and is based on the ideographs of Chinese writing. Finally, PCS (Picture Communication Symbols) was developed by Roxana Mayer-Johnson and today it is one of the most widely used thanks to its transparency level.

\section{Design of a Pictogram-Based Instant Messaging Service}

This section presents the design of Messenger Visual, an IM service that uses pictograms as the main communication system. Like any other IM service, Messenger Visual shall support all the basic IM features [8], such as creating user accounts and logging in to the service, as well as adding/removing contacts, sending/receiving presence updates and sending/receiving messages to/from users in the contact list. To implement the basic IM features of Messenger Visual we use XMPP (eXtensible Messaging and Presence Protocol) [11] because it is based on standards and provides a decentralized client-server architecture that is flexible, scalable and secure. But the fact that Messenger Visual has to support pictograms as the main communication system adds additional requirements to the IM service. These requirements, together with the methodology to integrate them into the IM service, are described below.

The first requirement of a pictogram-based IM service is that users shall have a pictogram set available to communicate. To support such requirement there are two alternatives; having a personalized set of pictograms for each user or having a pictogram set that is shared among all users. The former approach allows pedagogues to define exactly which pictograms each user should have and to tailor it according to 
their learning process, whereas the latter approach gives more freedom to the user as they have the chance to access all the pictograms to communicate. Nevertheless, both approaches have drawbacks. On the one hand, having a personalized pictogram set may limit conversations between users as specific pictograms might not be available to some individuals. On the other hand, including a complete pictogram set may have a negative impact on interactivity, as users might need to spend more time browsing for pictograms to compose a message. But considering that actual trends in pictogram-based communication state that AAC (Augmentative and Alternative Communications) users should have a full set of pictograms available to communicate in order to promote their independence and support their learning process we decided, together with pedagogues, to have a shared pictogram set among all users.

Taking into account that pedagogues should be able to select the pictographic system that is most suitable to users according to their personal preferences and previous knowledge, the second requirement of a pictogram-based IM service is that it shall remain independent of the pictographic system that users employ to communicate. Furthermore, considering that elements within the pictographic system include both graphical and textual representation of the pictogram itself, the IM service shall also remain independent of the textual representation of such pictograms. To satisfy this design requirement we have developed an XML-based syntax that is able to represent pictogram-based messages unequivocally. Despite the fact that both XML and JSON (JavaScript Object Notation) are platform and language neutral, we have decided to use the former instead of the latter because it has better support for internationalization, i.e. textual representation of Chinese characters, and parsers are readily available. The XML syntax, shown in Figure 1, defines that each pictogrambased message is represented by a message element, which may contain one or more pictogram elements to represent pictograms within the message.

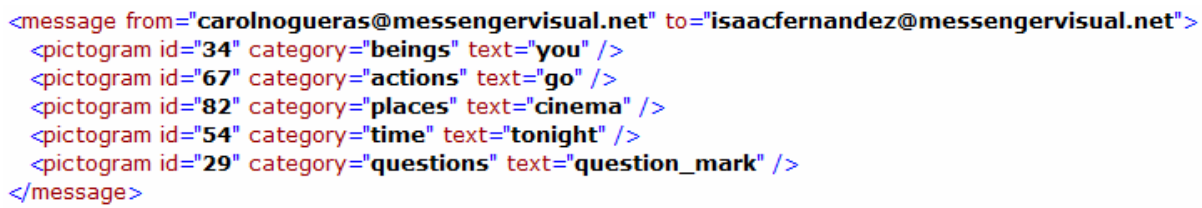

Fig. 1. Pictogram-based message representation using XML. A message element has a source and a destination and is composed of one or more pictogram elements. Each pictogram element contains a unique identifier, as well as the category it belongs to and the text associated to it.

Finally, considering that the vocabulary included in the pictogram set may change over time, the third requirement of a pictogram-based IM service is that it shall provide pedagogues with means for updating it. Two alternatives have been considered regarding the architecture to enable automatic pictogram updates; a centralized and a distributed approach. In the centralized architecture the pictogram set is stored in a server and retrieved by IM clients on demand. In contrast, in the distributed architecture each IM client has a local copy of the pictogram set that is synchronized with the server. In spite of requiring an additional synchronization mechanism, as pictogram set updates need to be notified to IM clients, we have decided to use a distributed architecture because it reduces the network bandwidth 
requirements as pictograms are cached at the IM client. To implement the pictogram set we use a relational database instead of separate files organized into folders because it is easier to maintain and offers performance advantages [12]. Finally, the protocol to enable pictogram database synchronization is based on XMPP to distribute update notifications among IM clients and HTTP (HyperText Transfer Protocol) to retrieve the pictogram database from the server, as represented in Figure 2.

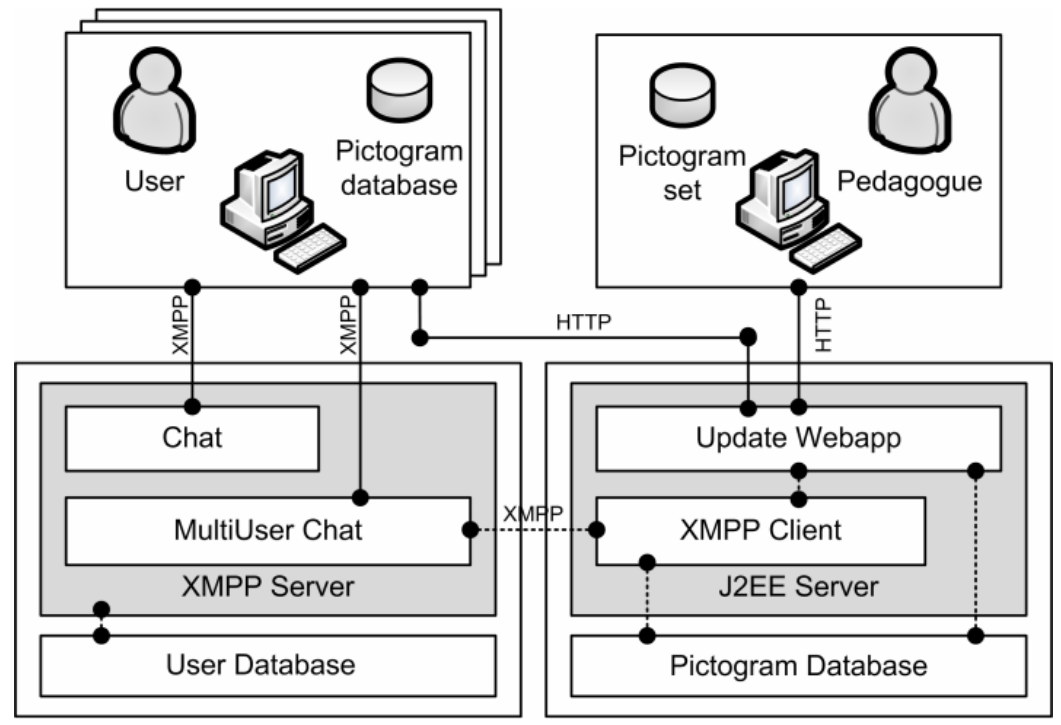

Fig. 2. Messenger Visual service architecture. XMPP supports the basic IM features, as well as pictogram-based communications and pictogram database update notifications. Whenever an update is triggered, IM clients download the pictogram database from the server using HTTP.

\section{Design of a Pictogram-Based Instant Messaging Client}

The next step to enable individuals with cognitive disabilities to communicate using the pictogram-based IM service is to develop an IM client that meets pictogram-based communication requirements. The IM client must provide a user interface that suits user requirements. As a first step, the requirements for a standard IM client were analyzed. In this sense, we found that the user interface of an IM client is organized to provide with a series of basic functionalities, such as access control, contacts management and chat conversations [8]. Although a first prototype of the IM client had already been implemented [13], it didn't completely meet the requirements of cognitively disabled users and, thus, required further analysis and development.

From this analysis we decided that the user interface should be organized with the same structure as a common IM client. Taking end user capabilities into account, the user interface should also be limited only to the strictly necessary elements to carry out the actions that users can perform in an IM service. Moreover, the user interface should be designed to be as simple as possible, avoiding the use of complex metaphors and 
without including hidden or implicit elements. In this sense, the user interface should be an almost completely visual interface. Written language might be included, but only as a support for social educators and users with some written language communication skills. Furthermore, elements in the user interface should be organized according to the requirements of the pictographic system, i.e. categories.

Albeit the IM service has been designed to remain independent from the pictographic system employed by users to communicate, we have initially decided to use a specific pictogram set as the IM client is to be evaluated with users at Fundació El Maresme, a non-profit organization that intends to promote the social integration of users with intellectual or cognitive disability and their families. To decide which set of pictograms should be used, we considered that users are already familiar with PCS (Picture Communication Symbols) because they use a piece of software called Boardmaker [14] in their daily activities. But considering the fact that PCS pictograms are licensed, and taking into account that ARASAAC (ARAgoneSe portal of Augmentative and Alternative Communication) pictograms [15] are similar but licensed under a Creative Commons BY-NC-SA license, we have finally decided to implement ARASAAC as the pictographic system of Messenger Visual.

The design of the IM client using a user-centered approach with users from Fundació El Maresme was conducted in fortnightly one-hour-long sessions that lasted for six months. During the sessions users were located in different rooms to resemble physical distance and pedagogues and social educators were present. These sessions served to evaluate the IM client in order to provide feedback about its possible shortcomings. After some iteration in the user interface design process, a first fully functional prototype that met all of the abovementioned requirements was developed.

To enforce access control the IM client is based on two different windows, as shown in Figure 3. First of all, the login window allows users to select their profile from a matrix of local user profiles stored in the computer. The window also offers a mechanism to create a new user account or to add an existing account to the local user profiles. Once users have selected their profile they must provide their passphrase in the password window to log in. Considering usability requirements the passphrase is based on a combination of four numeric digits represented by pictograms.

Once users have logged in, a window to manage contacts appears. The window contains a list with all the contacts that are currently online and is updated whenever a contact logs in or logs out. Taking into account usability requirements, the contacts in the list are represented by the user picture and full name. The contact window also provides a mechanism to add and remove contacts from the list. To start a new conversation with a contact from the list a user has to click on the contact picture.

Last but not least, the chat window allows users to communicate by means of exchanging pictogram-based messages. According to the ARASAAC pictogram organization, the top of the interface contains the categories in which pictograms are classified. On the left side the most frequent pictograms appear in order to ease the composition of common messages (i.e. Hello, Goodbye, Yes, No and Thank you). On the right side the pictograms that belong to the active category are displayed. On the bottom lies the pictogram input space to compose a message. Finally, the central part of the window contains the actual conversation that the user is having. 

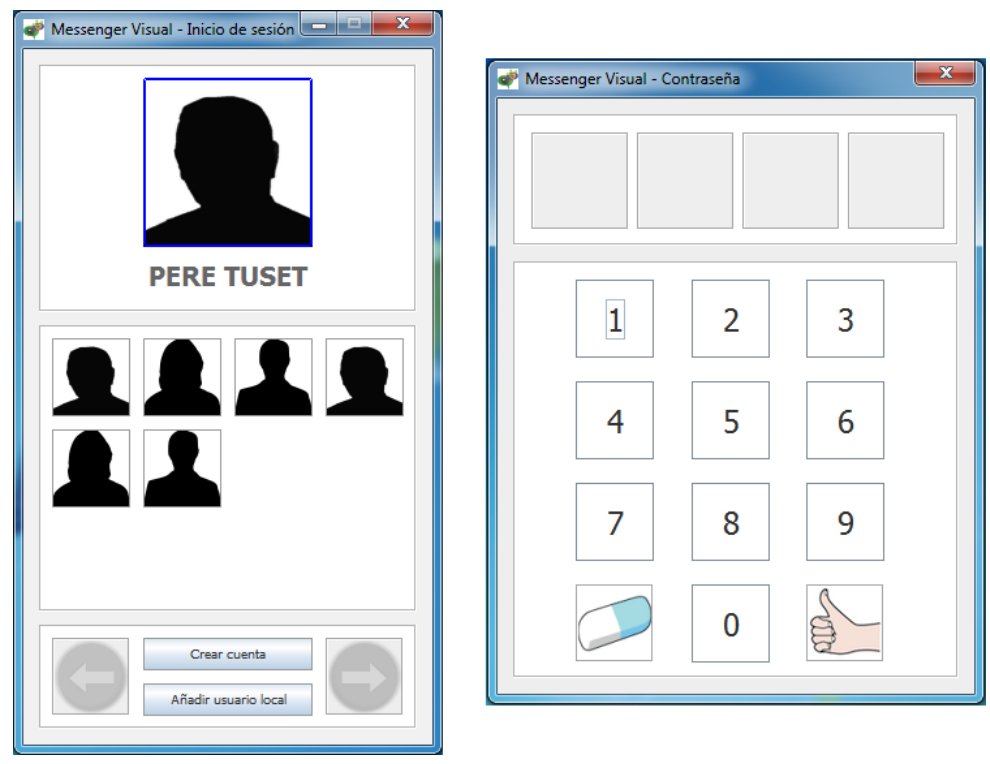

Fig. 3. Messenger Visual client user interface. The login window enables individuals to select their profile using a picture from the local user profile matrix, whereas the password window enables users to type in their four-digit numeric passphrase using pictograms.

\section{Conclusions and Future Work}

This paper has presented Messenger Visual, an IM service that enables individuals with cognitive disability to communicate over the Internet using pictogram-based messages. Along the text we have described and discussed the decisions to make Messenger Visual support the basic requirements of an IM service, as well as how we have enabled support for pictogram-based communications. We have also presented an IM client adapted to the requirements of individuals with cognitive disability and we have evaluated it using a user-centric approach. The evaluation has provided feedback that indicates that cognitively disabled individuals are able to communicate using pictograms, thus enabling social interaction and promoting digital inclusion.

Nevertheless, during the evaluation we have detected that some parts of the IM client user interface might need to be changed in order to increase usability. Some elements present a functionality that can be interpreted in different ways, thus becoming a source of possible confusion to users. For instance, buttons to navigate the contact list at the contact window operate in an up and down fashion, whereas buttons to navigate categories and pictograms in the chat window operate in a left and right fashion. Despite we have not observed important difficulties using such interface elements during the evaluation, we believe that redesigning those elements to provide more consistency will lead to an interface that is more usable by individuals.

Finally, all the knowledge we have today about how individuals with cognitive disability use Messenger Visual is based on qualitative research methods, such as interviews and ethnography. Thus, we are developing a tool to automatically collect 
and process statistical information about how users interact with the IM client user interface to communicate. For instance, we plan to explore parameters such as the average number of pictograms per message or the percentage of pictograms that users require in a conversation. This information, collected over time, will provide a deeper understanding of how users with cognitive disability communicate using pictograms.

\section{References}

1. World Health Organization. World report on disability, http://www.who.int/disabilities/world_report/concept_note_ 2010.pdf

2. Atkinson, R., Castro, D.: Digital Quality of Life: Understanding the Personal and Social Benefits from the Information Technology Revolution. In: Information Technology and Innovation Foundation (October 2008)

3. Glinert, E., York, B.: Computers and People with Disabilities. ACM Transactions on Accessible Computing 1(2) (October 2008)

4. LoPrestri, E., Bodine, C., Lewis, C.: Assistive Technology for Cognition: Understanding the Needs of Persons with Disabilities. IEEE Engineering in Medicine and Biology Magazine 27(2), 29-39 (2008)

5. Gregor, P., Dickinson, A.: Cognitive difficulties and access to information systems: an interaction design perspective. Journal of Universal Access to Information Society 5, $393-$ 400 (2007)

6. Todis, B., Sohlberg, M., Hood, D., Fickas, S.: Making electronic mail accessible: Perspectives of people with acquired cognitive impairments, caregivers and professionals. Brain Injury Journal 19(6), 389-401 (2005)

7. Poulson, D., Nicolle, C.: Making the Internet accessible for people with cognitive and communication impairments. Universal Access Information Society 3(1), 48-56 (2004)

8. Jennings, R., Nahum, E., Olshefski, D., Saha, D., Shae, Z., Waters, C.: A Study of Internet Instant Messaging and Chat Protocols. IEEE Network, 16-21 (July/August 2006)

9. Saint-Andre, P.: Streaming XML with Jabber/XMPP. IEEE Internet Computing, 82-89 (September/October 2005)

10. Glennen, S., DeCoste, D.: Handbook of Augmentative and Alternative Communication. Singular Publishing Group, San Diego (1998)

11. IETF. Extensible Messaging and Presence Protocol: Core (RFC3920), http: //tools.ietf.org/pdf/rfc3920.pdf (last accessed on January 7, 2011)

12. Sears, R., van Ingen, C., Gray, J.: To BLOB or Not To BLOB: Large Object Storage in a Database or a Filesystem? Technical Report (June 2006)

13. Tuset, P., Barberán, P., Janer, L., Delgado, S., Buscà, E., Vilà, N.: Messenger Visual: A pictogram-based IM service to improve communications among disabled people. In: NordiCHI 2010: 6th Nordic Conference on Human-Computer Interaction, pp. 797-800 (October 2010)

14. Mayer-Johnson: Boardmaker Software Family, http: / / www . mayer-johnson. com/products/boardmaker-software/

15. Aragonese Portal of Augmentative and Alternative Communication (ARASAAC), http: / / www. catedu.es/arasaac/ 